\title{
Numerical Prediction of Grid Erosion of Ion Engine
}

\author{
By Takeshi MiYASAKA ${ }^{1)}$, Tsutomu KobAYAshi ${ }^{1)}$ and Katsuo AsAto ${ }^{1)}$ \\ ${ }^{1)}$ Department of Human and Information Systems, Gifu University, Gifu, Japan
}

(Received July 17th, 2009)

With the increase of long-term space missions, the evaluation of lifetime of ion engines by numerical analyses becomes important. In order to develop a numerical code for the evaluation of ion engine lifetime, JIEDI (JAXA Ion Engine Development Initiative) tool development has been started. To evaluate the validities of boundary conditions such as upstream discharge region and downstream region conditions, a 3-dimensional full-particle code was developed. In the present study, the effects of the electron mass model introduced for shortening the calculation time were investigated. We found that there are differences in the distributions of charged particles and electric potential profiles in the downstream region among different electron masses. Consequently, the effects of electron mass on the energy peak of the ions impacting on the grid and the erosion distribution on the downstream surface of the accel grid were observed.

Key Words: Electric Propulsion, Ion Engine, Grid Erosion, PIC Code, JIEDI

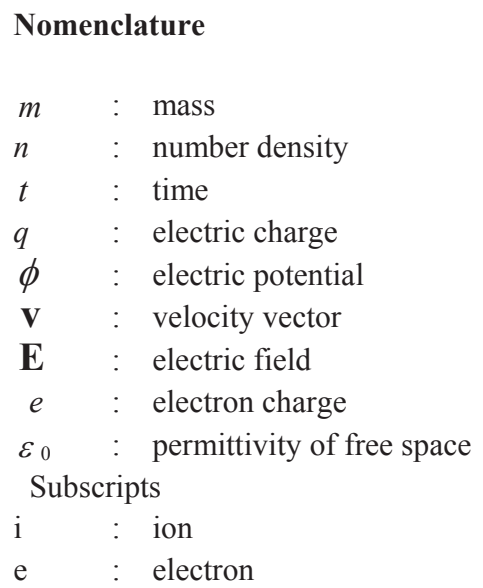

\section{Introduction}

A study of the ion engine lifetime is very important to enable its widespread application ${ }^{1)}$. Real time evaluations of the lifetime using test engines become difficult because the operation time increases as their areas of application expand.

A project of JIEDI (JAXA Ion Engine Development Initiatives) has been started for the development of a numerical tool for the evaluations of ion engine lifetime ${ }^{2)}$.

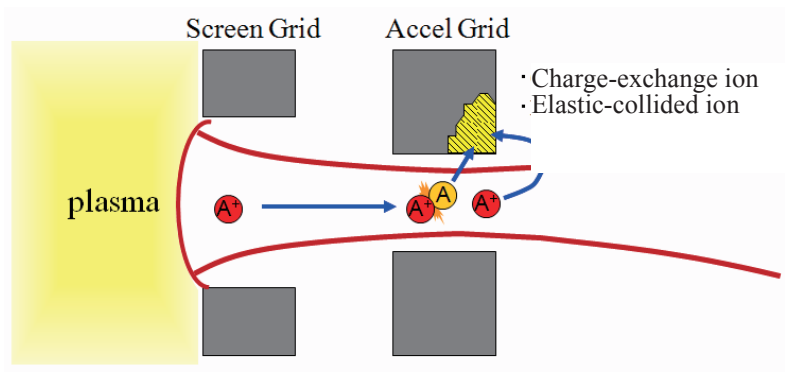

Fig. 1. Schematic of accel grid erosion.
Numerical simulations of ion engines have been performed by numerous workers ${ }^{3-9)}$ and the ion engine performance was predicted well. Lifetime evaluations have also been done numerically. The codes used for the evaluations are classified into the codes employing flux tubes to describe the motion of ions that constitute an ion beam and the particle codes treating the ions as particles. For quantitative evaluations of the lifetime of ion engines, three-dimensional analyses are required to consider the effects of multihole grids. However, the 3-dimensional analysis requires a very high calculation cost. The JIEDI tool is being developed on the basis of the flux-tube code by Nakano et al ${ }^{3-5)}$. The code employs the Boltzmann equation for describing distributions of electron number density. Because of the electron model, analyses by the code have a short calculation time. Accordingly, the JIEDI tool based on the flux-tube code has some models. For further quantitative evaluations of the lifetime, investigations of the effect of the models on the results of the analyses are required. For these reasons, particle analyses of the lifetime have also been started. A full-PIC (particle in cell) code is employed in the analyses. The full-PIC code treats both ions and electrons as particles. However, the analyses require an enormous amount of time because of the light electron mass. Therefore, 3 -dimensional analyses using the real electron mass are not realistic. Consequently, in the 3-dimensional analyses, the introduction of an artificial electron mass heavier than the real mass is required.

In the present paper, we describe the results of particle analyses for various artificial electron masses and discuss the effects of the artificial electron mass on the particles and electric potential distributions and on the ions impacting on the grid, which cause grid erosion, as shown in Fig. 1.

\section{Numerical Analysis}

Analyses of charged particles were performed for the

Copyright $\odot 2010$ by the Japan Society for Aeronautical and Space Sciences and ISTS. All rights reserved. 
two-grid ion engine used in the experiment by Hayakawa ${ }^{10,11)}$. The ion engine has seven holes on the grids. However, to shorten the calculation time, the calculation domain is minimized, as shown in Fig. 2. On the boundary surfaces, symmetric conditions are applied, as shown in Fig. 3. The numerical procedures are summarized in Fig. 4. Specific procedures are explained in detail below.

\subsection{Charged particle calculations}

Charged particle motions are calculated by a full-PIC method ${ }^{12)}$. In the analyses, xenon is employed as a propellant. Both ions and electrons are treated as particles, and they move according to the following equation of motion:

$$
m_{\mathrm{p}} \frac{d \mathbf{v}_{\mathrm{p}}}{d t}=q_{\mathrm{p}} \mathbf{E}
$$

where subscript $p$ in Eq. (1) represents $i$ or e. For higher time

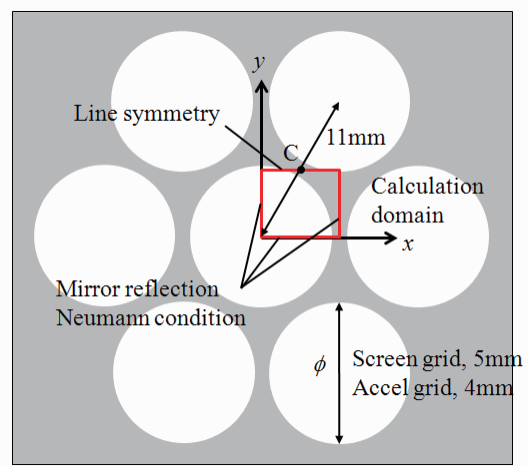

Fig. 2. Schematic of 7-hole grid and calculation domain and conditions.

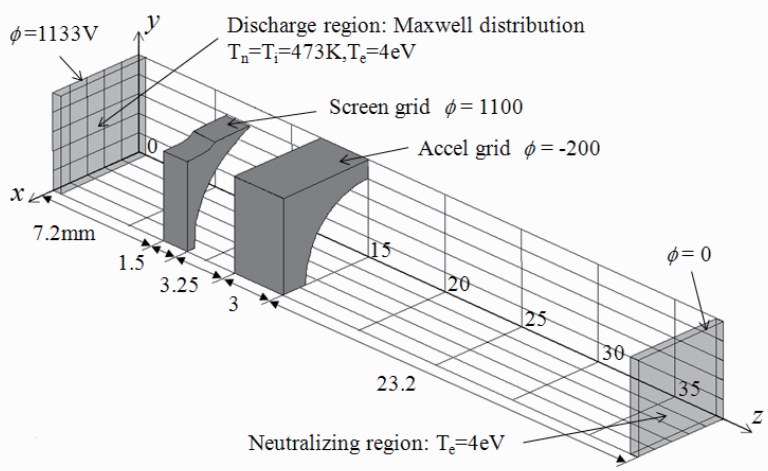

Fig. 3. Schematic of 3-dimensional calculation domain and conditions.

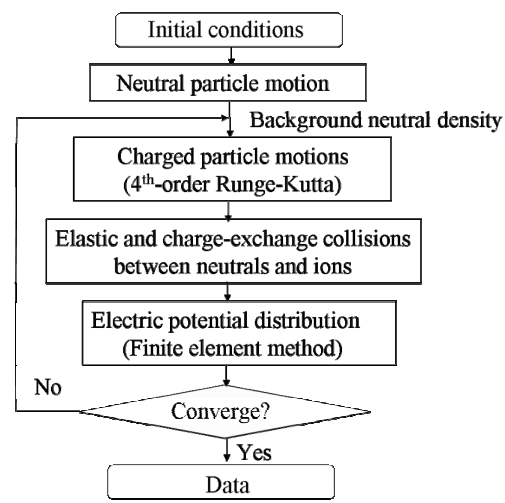

Fig. 4. Schematic of numerical procedure. accuracy, the $4^{\text {th }}$-order Runge-Kutta method is employed for the time integration of Eq. (1).

In order to shorten the calculation time, an electron model is introduced into the analyses. The electron model treats electron masses heavier than the real mass. However, in the case of using the electron model, the influence of the model on the results should be investigated. Therefore, in the present analyses, various electron masses are applied for comparison.

Collisions between ions and neutrals are calculated for the evaluation of collided particle impact on the accel grid. In the present study, the charge-exchange and elastic collisions are calculated. The collision calculation requires the neutral density distribution. The neutral distribution is obtained from the neutral calculations by DSMC (direct simulation of Monte Carlo) in advance. The neutral calculation does not treat collisions between neutrals because of the larger Knudsen number.

As shown in Fig. 2, the conditions of mirror reflection are given on the surfaces at $x$ and $y$ boundaries, except $y=y_{\max }$. On the surface at $y=y_{\max }$, a condition of line symmetry concerning line $\mathrm{C}$ is applied. In the upstream boundary region, ions and electrons with the Maxwell distribution are given uniformly. In addition, uniform neutralizing electrons are supplied to the downstream region. The ion number density in the upstream boundary region is adjusted for the given value of the beamlet current. The electron number density in the region is the same as that of ions. The number density of the neutralizing electron is determined from the ion number density distribution at the exit plane. The density is set to the average value of the ion densities of the beamlet. The temperatures of the charged particles obtained from the experiment are given in the boundary regions.

\subsection{Electric potential calculation}

Electric potential distributions are obtained by solving the Poisson equation by the FEM method.

$$
\Delta \phi=-\frac{e\left(n_{\mathrm{i}}-n_{\mathrm{e}}\right)}{\varepsilon_{0}}
$$

On the surfaces at $x$ and $y$ boundaries except $y=y_{\max }$, the Neumann conditions are applied. As in the particle calculations described above, the boundary condition on the surface at $y=y_{\max }$ is treated with the line symmetry concerning the line C. On the screen and accel grid boundaries, the potential of grid points not on the electrode surfaces are estimated by extrapolation. In the numerical simulations of ion engines, some different conditions on the exit boundary surface are used. Because uniform electrons are set in the downstream region in the present analyses, the uniform electric potential of $0 \mathrm{~V}$ is applied on the boundary surface.

\subsection{Grid system and numerical condition}

The present analyses employ a rectangular grid. The number of gird points is 43,39 , and 160 in the $x, y$, and $z$ directions, respectively. Grid size is not uniform in the z-direction. In the discharge and neutralizing regions, the grid sizes are decided on the basis of the respective Debye lengths. The time interval in the time integration of Eq. (1) is selected to satisfy the following condition on the plasma oscillation.

$$
\Delta t<\frac{1}{\omega_{\mathrm{p}}}
$$




\section{Results and Discussion}

To evaluate the effects of the electron mass model, analyses with four different electron masses heavier than the real mass were performed. The mass ratios of the ion mass to the electron masses used in the analyses are 10, 100, 1000, and 4000. Below are the results of the comparison of mass ratio effects on the distributions of charged particles and electric potential and on the properties of ion impact on the accel grid, which cause grid erosion.

The calculations are performed using Real Computing Opteron shanghai 2376 (AMD $2.3 \mathrm{GHz}$ ) and 2380 (AMD 2.5 $\mathrm{GHz}$ ). It takes about 24 hours to get the converged distributions in each case. However, to obtain the data of the same number of impacting ions on the accel grid surface, the computational time increases roughly in proportion to the square root of the mass ratio.

\subsection{Calculated distributions for the mass ratio of $\mathbf{1 0 0 0}$}

As examples of calculated distributions, the 3-dimensional particle distributions in the case of the mass ratio of 1000 are shown in Figs. 5 and 6. Figure 5 shows the distributions of ion and electron particles. In Fig. 5, an ion beam profile and areas where electrons exist in the upstream and downstream regions are observed. In addition to the ions constituting the beam, ions straying from the beam are observed in the figure. Figure 6 shows the straying ions observed in Fig. 5, by type. In the upstream region, most of the straying ions are elastic-collision ions. In the holes and downstream region, three types of ions, elastic collision, charge-exchanged, and multicollision ions are observed. In the region outside the ion beam, the charge-

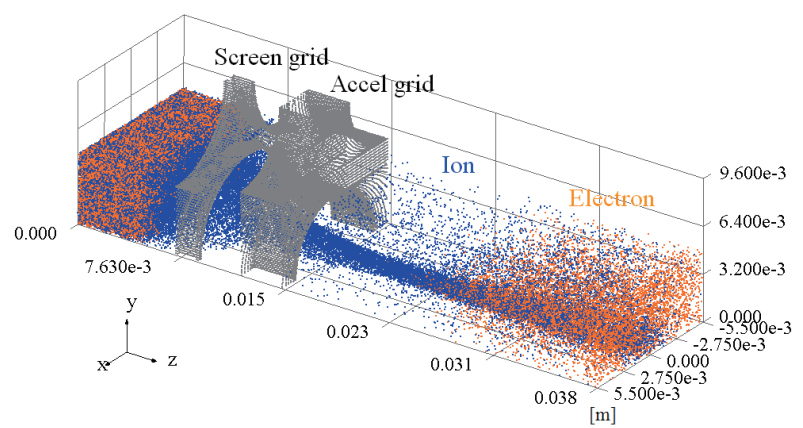

Fig. 5. Three-dimensional distributions of ion and electron particles for mass ratio of 1000 .

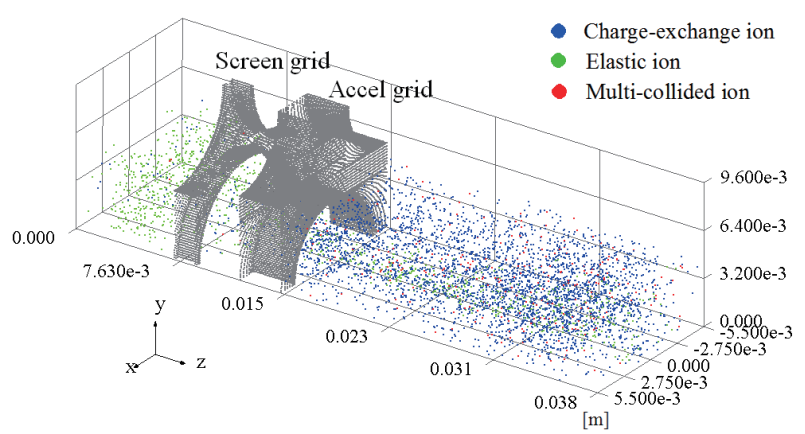

Fig. 6. Three-dimensional distributions of collided ions for mass ratio of 1000 . exchanged and multicollision ions exist. Some of the stray ions in the region impact the grids and cause grid erosion.

3.2. Effects of mass ratio on distributions of charged particles and electric potential

To evaluate the effects of the mass ratio, the results in the cases of the mass ratios of 10, 100, 1000, and 4000 are compared. The calculated beamlet currents are shown in Table 1. The beamlet current for the mass ratio of 10 is significantly high compared with the others. However, among the mass ratios of 100,1000 , and 4000 , the difference is within several percent.

Figures 7 and 8 show the 2-dimensional distributions of the ion and electron particles at $y=0 \mathrm{~mm}$ for the four mass ratios. These figures do not show much difference among the four

Table 1. Calculated beamlet current.

\begin{tabular}{|c|c|c|c|}
\hline case & $\mathrm{m}_{\mathrm{i}} / \mathrm{m}_{\mathrm{e}}$ & $\mathrm{m}_{\mathrm{e}} /\left[\right.$ real $\left.\mathrm{m}_{\mathrm{e}}\right]$ & beamlet current $[\mu \mathrm{A}]$ \\
\hline (a) & 10 & 23943 & 90.7 \\
\hline (b) & 100 & 2394 & 79.6 \\
\hline (c) & 1000 & 239 & 80.5 \\
\hline (d) & 4000 & 60 & 80.4 \\
\hline Real & 239433 & 1 & - \\
\hline
\end{tabular}

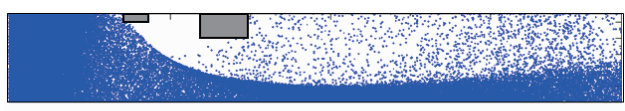

(a) $\mathrm{m}_{\mathrm{i}} / \mathrm{m}_{\mathrm{e}}=10$

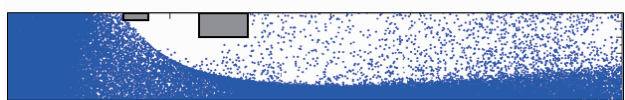

(b) $\mathrm{m}_{\mathrm{i}} / \mathrm{m}_{\mathrm{e}}=100$

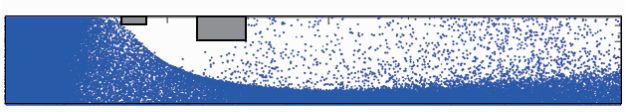

(c) $\mathrm{m}_{\mathrm{i}} / \mathrm{m}_{\mathrm{e}}=1000$

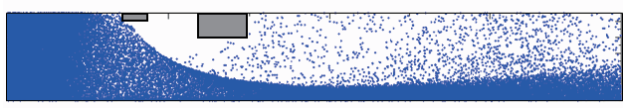

(d) $\mathrm{m}_{\mathrm{i}} / \mathrm{m}_{\mathrm{e}}=4000$

Fig. 7. Two-dimensional distributions of ion particles for various mass ratios $(y=0)$.

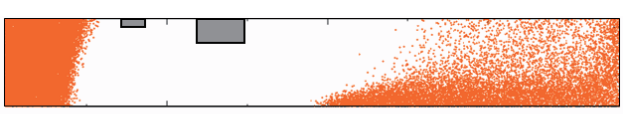

(a) $\mathrm{m}_{\mathrm{i}} / \mathrm{m}_{\mathrm{e}}=10$

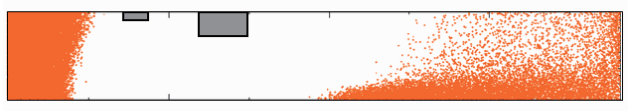

(b) $\mathrm{m}_{\mathrm{i}} / \mathrm{m}_{\mathrm{e}}=100$
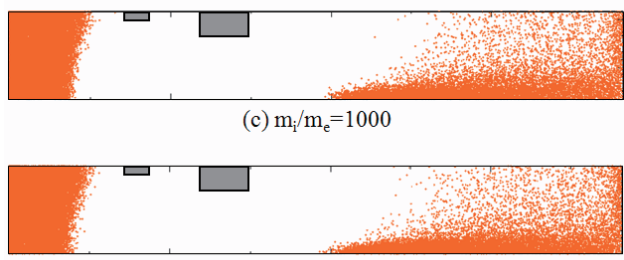

(d) $\mathrm{m}_{\mathrm{i}} / \mathrm{m}_{\mathrm{e}}=4000$

Fig. 8. Two-dimensional distributions of electron particles for various mass ratios $(y=0)$. 


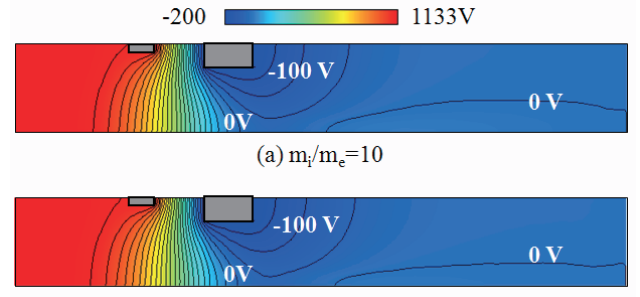

(b) $\mathrm{m}_{\mathrm{i}} / \mathrm{m}_{\mathrm{e}}=100$

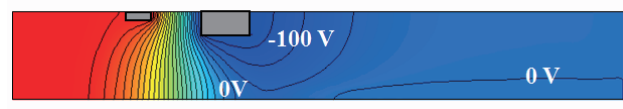

(c) $\mathrm{m}_{\mathrm{i}} / \mathrm{m}_{\mathrm{e}}=1000$

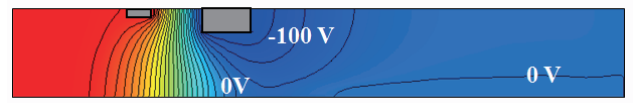

(d) $\mathrm{m}_{\mathrm{i}} / \mathrm{m}_{\mathrm{e}}=4000$

Fig. 9. Two-dimensional distributions of electric potential for various mass ratios $(y=0)$.

profiles. However, in the downstream region, the existence areas of the ions constituting the beamlet and neutralizing electrons for the mass ratio of 10 are slightly wider compared with the others. In the electric potential distributions shown in Fig. 9, similar tendencies are also observed. In the downstream region, there is a slight difference in the electric potential contour profiles between the cases of the mass ratio of 10 and the others. The difference in the existence areas of the neutralizing electrons cause the difference in the positive areas of the electric potential. Because of the different distribution of the electric potential, the existing areas of the ion beamlet for the mass ratio of 10 are slightly different from the others.

To observe the difference in detail, 1-dimensional distributions are plotted in Figs. 10-13. Figures 10 and 11 show axial distributions of the charged particles and electric potential around the sheath region along the line close to the centerline of the holes at $x=0.5 \mathrm{~mm}$ and $y=0 \mathrm{~mm}$. In Fig. 10, the sheath profiles of the ions and electrons for the mass ratio of 10 show slight differences compared with the other ratios. Because of the effect of the electron particle distributions, the electric potential distribution shown in Fig. 11 also shows similar tendencies.

Figures 12 and 13 show radial distributions of the charged particles and electric potential in the downstream region along the line at $z=25 \mathrm{~mm}$ and $y=0 \mathrm{~mm}$. In Fig. 12, the ion and electron profiles for the lower mass ratios of 10 and 100 show significant differences compared with those for the higher ratios of 1000 and 4000. As a result, in Fig. 13, the electric potential for the lower mass ratios of 10 and 100 are obviously higher than those for higher ratios. For the high mass ratios, the velocities of the electrons are sufficiently high compared with those of the ions. Thus, the mass ratio has an insignificant effect on the electron velocities. However, for the low mass ratios, the velocities of the electron are comparable with those of the ions. Therefore, for the low mass ratios, the mass ratio affects the electron particle distributions. The dependency of the electron motion on the mass ratio causes the differences of the electron potential distribution.

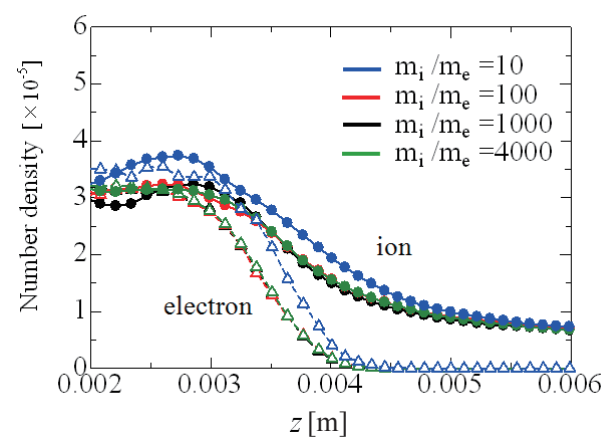

Fig. 10. Distributions of ion and electron number densities in the $z$ direction around the sheath region at $x=0.5 \mathrm{~mm}$ and $y=0 \mathrm{~mm}$ for various mass ratios.

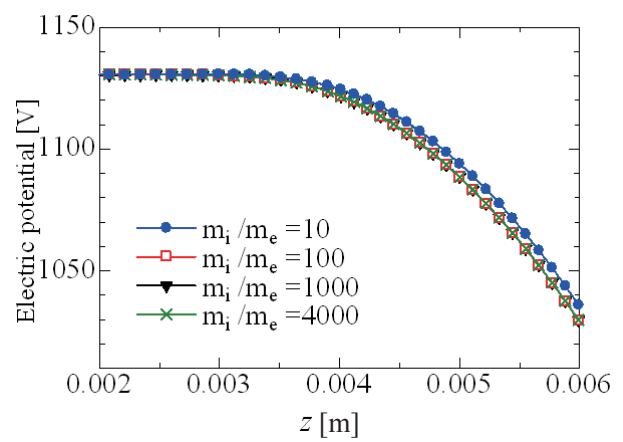

Fig. 11. Distributions of electric potential in the $z$ - direction around the sheath region at $x=0.5 \mathrm{~mm}$ and $y=0 \mathrm{~mm}$ for various mass ratios.

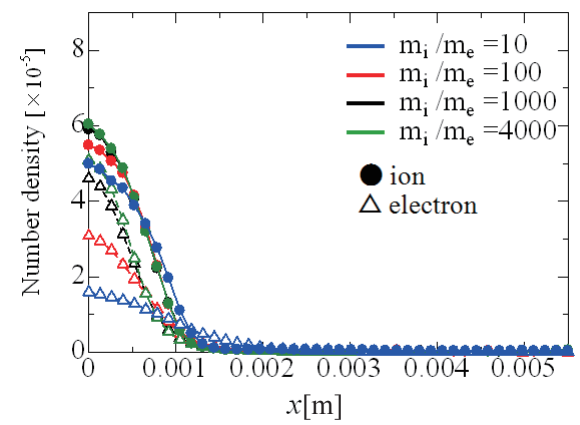

Fig. 12. Distributions of ion and electron number densities in the $x$ direction in the downstream region at $z=25 \mathrm{~mm}$ and $y=0 \mathrm{~mm}$ for various mass ratios.

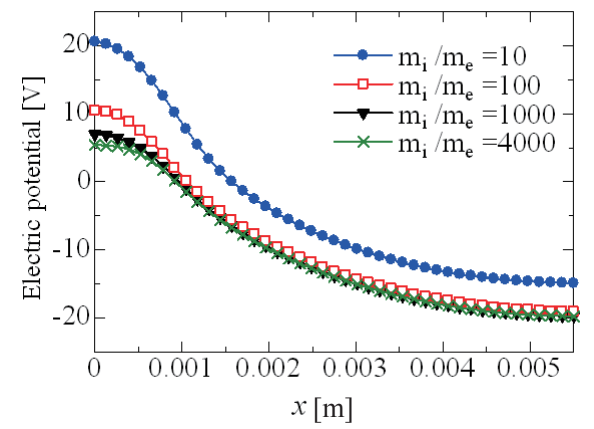

Fig. 13. Distributions of electric potential in the $x$-direction in the downstream region at $z=25 \mathrm{~mm}$ and $y=0 \mathrm{~mm}$ for various mass ratios. 
Consequently, as shown in Figs. 7 and 12, the ion beamlet expands in the radial direction for the mass ratio of 10 .

These results indicate the existence of the effects of mass ratios lower than 100. In addition, there are no significant differences between the results for higher mass ratios of 1000 and 4000. Therefore, to achieve reasonable distributions of charged particles and electric potential, an electron mass which gives a mass ratio higher than 1000 is required.

\subsection{Effects of mass ratio on accel grid erosion}

The main purpose of our project is to evaluate grid erosion. Thus, evaluations of grid currents and the properties of ions impacting on the grids are important.

Calculated ratios of accel current to beamlet current were compared with those from the experiment. The accel current was treated separately and divided into the currents to the upstream and inner surfaces and the current to the downstream surface, as shown in Fig. 14. Figure 15 shows the calculated ratios and experimental values. In Fig. 15, both ratios agree well with the experimental values. For both types of ratios, the difference among the four mass ratios is within $4 \%$.

Figure 16 shows the energy distributions of ions impacting on the downstream surface of the accel grid for various mass ratios. In each case, the total number of samples for this energy distribution is fixed. The energy distribution for each mass ratio has two peaks near 150 and $200 \mathrm{eV}$. As shown in

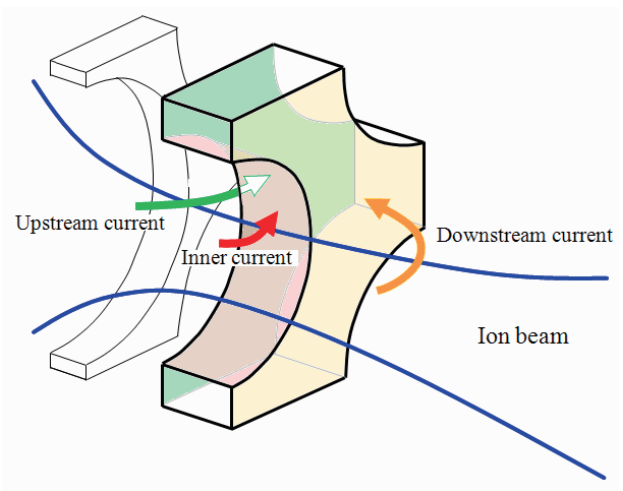

Fig. 14. Schematic of accel grid currents to three surfaces, the upstream surface, inner surface, and downstream surface.

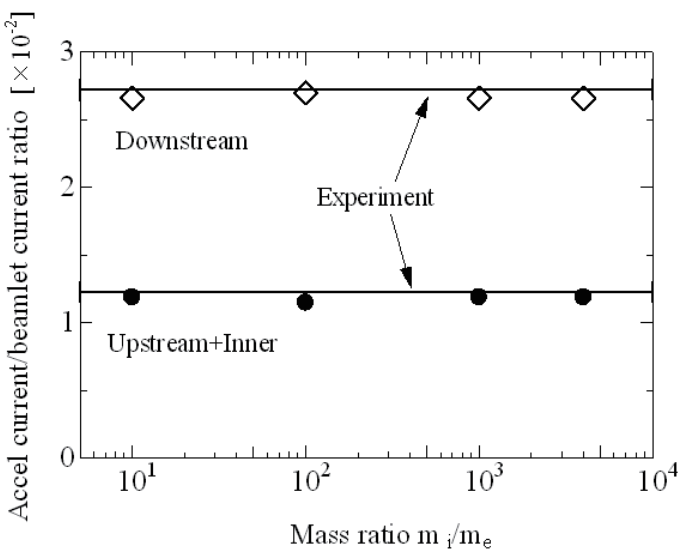

Fig. 15. Ratios of accel current to beamlet current calculated numerically and found in the experiment for various mass ratios.
Fig. 9, the electron potential distribution shows two possible areas in which the collided ions impacting downstream surface of the accel grid are generated, the positive potential area near the accel grid hole and the almost zero potential area in the downstream region. Thus, the energy distributions in Fig. 16 show the peak near the accel grid potential and that lower than the potential. However, the peak near $200 \mathrm{eV}$ for the mass ratio of 10 is significantly broader than the others. The wider positive region of the electric potential in the

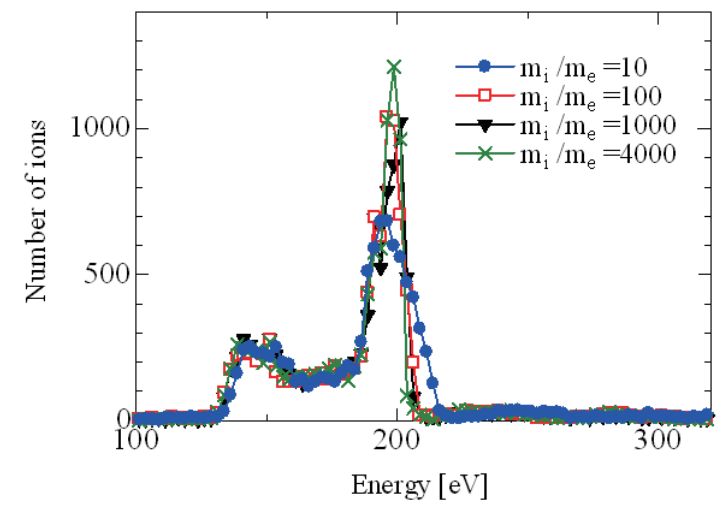

Fig. 16. Energy distributions of ions impacting on the downstream surface of the accel grid for various mass ratios.

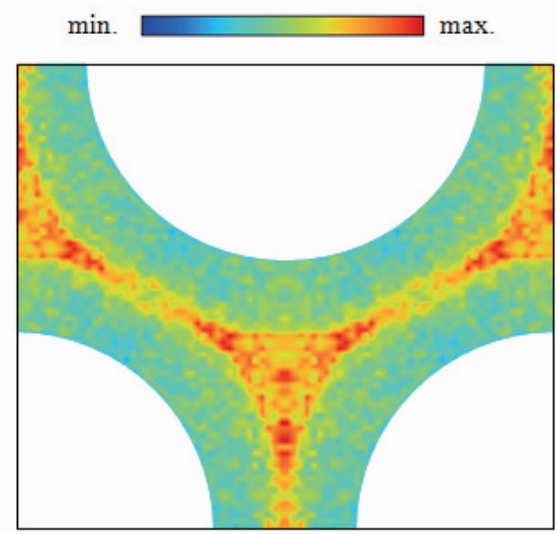

(a) $\mathrm{m}_{\mathrm{i}} / \mathrm{m}_{\mathrm{e}}=10$

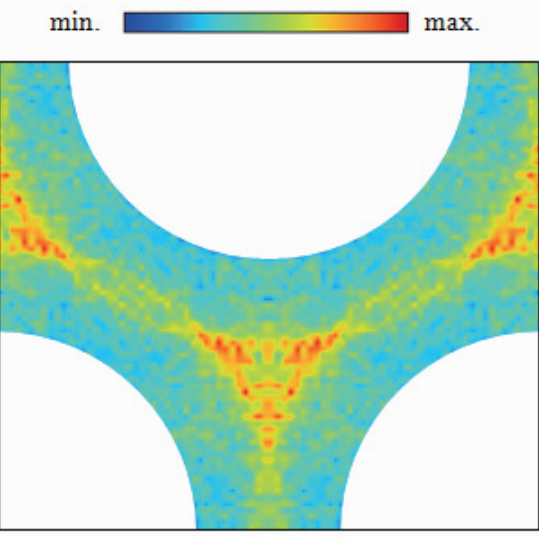

(c) $\mathrm{m}_{\mathrm{i}} / \mathrm{m}_{\mathrm{e}}=1000$

Fig. 17. Erosion distributions after ion impact on the downstream surface of the accel grid for mass ratios of 10 and 1000 . 


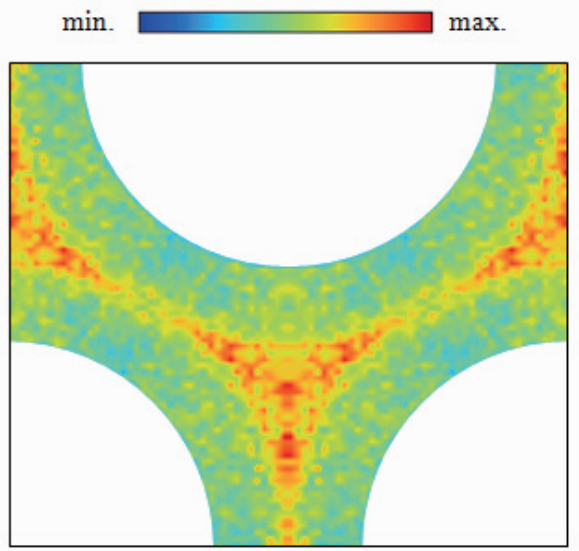

(a) $\mathrm{m}_{\mathrm{i}} / \mathrm{m}_{\mathrm{e}}=10$

$\min$

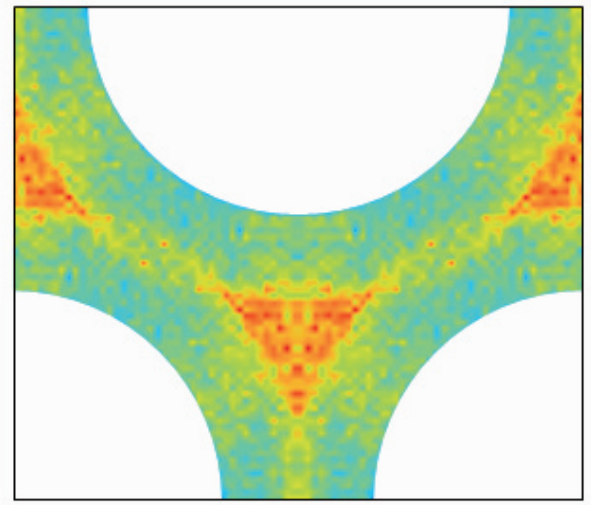

(c) $\mathrm{m}_{\mathrm{i}} / \mathrm{m}_{\mathrm{e}}=1000$

Fig. 18. Ion number flux density distributions to the downstream surface of the accel grid for mass ratios of 10 and 1000 .

downstream region for the mass ratio of 10 , as described in Sec.3.2, causes the broader peak. The energy distribution affects the erosion of the grid directly. Therefore, the results also indicate the importance of selecting the mass ratio for detailed quantitative evaluations of the grid erosion distributions.

Figure 17 shows the erosion distributions on the downstream surface of the accel grid for the mass ratios of 10 and 1000, which were estimated using the semi-empirical equation by Yamamura et al. ${ }^{13)}$. Each distribution shows a characteristic

erosion pattern called "pits \& grooves". The erosion for the higher mass ratio of 1000 is concentrated in the center region among the holes, compared with that for the lower mass ratio of 10. A similar difference to that in the erosion pattern between the higher and lower mass ratios is observed in the distributions of ion number flux density to the downstream surface of the accel grid, shown in Fig. 18. Thus, the result indicates that the difference in the erosion pattern is due to that in the ion flux density distribution.

\section{Conclusion}

In order to evaluate the effects of artificial electron mass on the evaluation of grid erosion in ion engines using a 3-dimensional full-PIC code, analyses were performed for four different mass ratios of ion mass to electron mass, 10, 100,1000 , and 4000. As a result, we found that the distributions of the charged particles and electric potential for the lower mass ratios of 10 and 100 are different from those for the higher mass ratios of 1000 and 4000. While the ratios of the accel grid currents to the beamlet current show little difference, the energy peak of the ions constituting the current becomes broader for the mass ratio of 10. Each erosion pattern of the downstream surface of the accel grid shows a pattern typical of a two-grid ion engine. However, the erosion pattern for the higher mass ratio is significantly different from that for the lower mass ratio.

These results indicate the basic effectiveness of the developed 3-dimensional full-PIC code and the necessity for the use of a higher mass ratio.

\section{Acknowledgments}

This work was supported by Japan Aerospace Exploration Agency under cooperative research project No. 501000402. The authors would like to thank Mr. T. Suzuki and members of the JIEDI project for their supports.

\section{References}

1) Arakawa, Y., Kuninaka, H., Nakayama, N. and Nishiyama, K.: Ion Engines for Powered Flight in Space, Corona Publishing, 2006 (in Japanese).

2) Kuninaka, H: Research and Development of JIEDI (JAXA Ion Engine Development Initiatives) Tool for Numerical Evaluation of Ion Engine Grid Lifetime, JAXA Special Publication, JAXA-SP-06-019, pp.5-9, 2007 (in Japanese).

3) Nakano, M. and Arakawa, Y.: Numerical Simulation of Ion Thruster Grid Lifetime, J. Jpn. Soc. for Aeronaut. Apace Sci, 48 (2000), pp.111-117 (in Japanese).

4) Nakano, M: Three-dimensional Simulations of Ion Engine Grid Erosion, Advances in Applied Plasma Science, 6 (2007), pp.93-96.

5) Nakano, M.: Improvements in the Grid Erosion Evaluation Code for Ion Engines, JAXA-SP-06-019, JAXA Special Publication, pp.47-53, 2007 (in Japanese).

6) Nakayama, N. and Wilbur, P.J.: Numerical Simulation of Ion Beam Optics for Multiple-grid Systems, J. Propulsion and Power, 19 (2003), pp.607-613.

7) Wang, J., Cao, Y., Kafafy, R., Martinez, R. and Williams, J.: AIAA paper 2006-4999, 2006.

8) Tartz, M., Hartmann, E., Deltschew, R. and Neumann, H.: Validation of a Grid Erosion Simulation by Short-time Erosion Measurements, Proceedings of the 26th International Electric Propulsion Conference, IEPC-99-147, 1999.

9) Okawa, Y., Takegahara, H.: Particle Simulation on Ion Beam Extraction Phenomena in an Ion Thruster, Proceedings of the 26th International Electric Propulsion Conference, IEPC-99-146, 1999.

10) Hayakawa, Y.: Measurements of Current Distribution on a Two-grid-ion-extraction-system Gridlet, Proceedings of 42nd Joint Propulsion Conference, AIAA-2006-5003, pp.1-9, 2006.

11) Hayakawa, Y.: Current Distribution on a Two-grid-ion-extraction-system Gridlet, JAXA Special Publication: JAXA-SP-06-019, pp.20-27, 2007 (in Japanese).

12) Birdsall, C.K. and Langdon, A.B.: Plasma Physics via Computer Simulation, MacGraw-Hill, 1985.

13) Hyakutake, T., Nishida, M., Kenmotsu, T. and Muramoto, T.: Toward Sputtering Analysis for Ion Engine Accelerator Grid, JAXA Special Publication, JAXA-SP-06-019, pp.59-63, 2007 (in Japanese). 